\title{
Structural basis for a naphthyl end-capped oligothiophene with embedded metallic nanoparticles for organic field-effect transistors
}

\author{
Huss-Hansen, Mathias Kasper; Hansteen, Marie; Linnet, Jes; Walther, Anders Runge; Kjelstrup-Hansen, \\ Jakob; Knaapila, Matti
}

Published in:

Applied Physics Letters

Link to article, DOI:

$10.1063 / 1.5080006$

Publication date:

2018

Document Version

Publisher's PDF, also known as Version of record

Link back to DTU Orbit

Citation (APA):

Huss-Hansen, M. K., Hansteen, M., Linnet, J., Walther, A. R., Kjelstrup-Hansen, J., \& Knaapila, M. (2018). Structural basis for a naphthyl end-capped oligothiophene with embedded metallic nanoparticles for organic field-effect transistors. Applied Physics Letters, 113(25), [251903]. https://doi.org/10.1063/1.5080006

\section{General rights}

Copyright and moral rights for the publications made accessible in the public portal are retained by the authors and/or other copyright owners and it is a condition of accessing publications that users recognise and abide by the legal requirements associated with these rights.

- Users may download and print one copy of any publication from the public portal for the purpose of private study or research.

- You may not further distribute the material or use it for any profit-making activity or commercial gain

- You may freely distribute the URL identifying the publication in the public portal 


\section{Structural basis for a naphthyl end-capped oligothiophene with embedded metallic nanoparticles for organic field-effect transistors}

Mathias K. Huss-Hansen, Marie Hansteen, Jes Linnet, Anders Runge Walther, Jakob Kjelstrup-Hansen, and Matti Knaapila

Citation: Appl. Phys. Lett. 113, 251903 (2018); doi: 10.1063/1.5080006

View online: https://doi.org/10.1063/1.5080006

View Table of Contents: http://aip.scitation.org/toc/apl/113/25

Published by the American Institute of Physics

\section{Articles you may be interested in}

Improved performance of InP-based $2.1 \mu \mathrm{m}$ InGaAsSb quantum well lasers using $\mathrm{Sb}$ as a surfactant Applied Physics Letters 113, 251101 (2018); 10.1063/1.5060653

Low temperature preparation of $\mathrm{HfO}_{2} / \mathrm{SiO}_{2}$ stack structure for interface dipole modulation Applied Physics Letters 113, 251601 (2018); 10.1063/1.5057398

A high-responsivity and broadband photoconductive terahertz detector based on a plasmonic nanocavity Applied Physics Letters 113, 251102 (2018); 10.1063/1.5066243

Impurity-derived p-type conductivity in cubic boron arsenide

Applied Physics Letters 113, 251902 (2018); 10.1063/1.5058134

Capillary-assisted localized crystallization on discrete micropillar rings

Applied Physics Letters 113, 251904 (2018); 10.1063/1.5063608

Enhanced response of bulk heterojunction polymer photodetectors upon incorporating $\mathrm{CsPbBr}_{3}$ quantum dots Applied Physics Letters 113, 253301 (2018); 10.1063/1.5050253

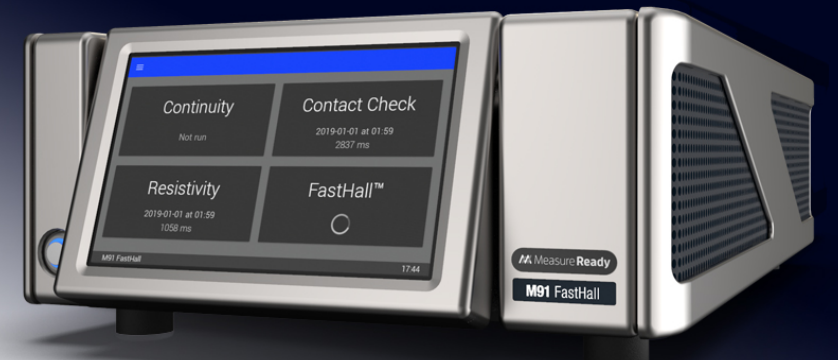

\section{Measure Ready M91 FastHall ${ }^{\mathrm{TM}}$ Controller \\ A revolutionary new instrument for complete Hall analysis}




\title{
Structural basis for a naphthyl end-capped oligothiophene with embedded metallic nanoparticles for organic field-effect transistors
}

\author{
Mathias K. Huss-Hansen, ${ }^{1, a)}$ Marie Hansteen, ${ }^{1}$ Jes Linnet, ${ }^{2,3, b)}$ Anders Runge Walther, ${ }^{2,4}$ \\ Jakob Kjelstrup-Hansen, ${ }^{2}$ and Matti Knaapila ${ }^{1}$ \\ ${ }^{1}$ Department of Physics, Technical University of Denmark, 2800 Kgs. Lyngby, Denmark \\ ${ }^{2}$ NanoSYD, Mads Clausen Institute, University of Southern Denmark, 6400 Sфnderborg, Denmark \\ ${ }^{3}$ The Marsk Mc-Kinney Moller Institute, University of Southern Denmark, 5230 Odense, Denmark \\ ${ }^{4}$ Department of Chemical Engineering, Biotechnology and Environmental Technology, University of Southern \\ Denmark, 5230 Odense, Denmark
}

(Received 5 November 2018; accepted 5 December 2018; published online 20 December 2018)

\begin{abstract}
We report on the apparent structure of $5,5^{\prime \prime}$-bis(naphth-2-yl)-2,2 $: 5^{\prime}, 2^{\prime \prime}$-terthiophene (NaT3) in organic field-effect transistors (OFETs) with and without embedded silver nanoparticles. Using regular- and microbeam grazing incidence wide- and small-angle X-ray scattering, the device structure is characterized locally in the area with the embedded particles. The NaT3 thin film order is reduced and the found unit cell $\left(a=25.7 \AA, b=5.87 \AA, c=8.03 \AA\right.$, and $\left.\beta=98.9^{\circ}\right)$ differs significantly from the one reported in the bulk, but shows no significant change, when the particles corresponding to the crystal size are incorporated into the device structure. At the same time, the apparent thin film crystal sizes in OFETs are found to be similar with and without the embedded particles. In both cases, the carrier mobilities are of the order of $10^{-4} \mathrm{~cm}^{2} /(\mathrm{V} \mathrm{s})$. Published by AIP Publishing. https://doi.org/10.1063/1.5080006
\end{abstract}

Organic field-effect transistors (OFETs) are subject to interest for use in modern electronics due to their inexpensive, low-temperature processing, low materials costs, and application potential in, e.g., flexible electronics, ${ }^{1}$ gas sensors, ${ }^{2}$ pressure sensors, ${ }^{3}$ and optical sensors (organic phototransistors, OPTs). ${ }^{4}$ Numerous studies have been carried out on conjugated oligomers and polymers, whose properties can be tuned through design and functionalization. ${ }^{5,6}$ Of particular interest are thiophene-based oligomers because of their hole transport properties and stable device performance under atmospheric conditions. ${ }^{7-9}$ By end-capping the thiophene rings with aryl groups, the polymerization potential is hindered and intermolecular packing is promoted. ${ }^{9}$

We have recently shown that the photoresponsivity in naphthyl end-capped oligothiophene based OPTs can be enhanced by utilizing the plasmonic properties of embedded metallic nanoparticles. ${ }^{10}$ However, while it is well known that the carrier mobility is connected to the orientation and crystal size of the organic molecules, ${ }^{11-13}$ the literature of structural evaluation of oligothiophenes in OFETs with embedded metallic particles remains less comprehensive.

The molecular structure of organic thin film devices is often evaluated by the use of grazing incidence X-ray scattering. ${ }^{14-16}$ OFETs with embedded nanoparticle arrays are prepared using electron-beam lithography, which does not allow for a simple preparation of suitably large samples for grazing-incidence studies. Therefore, it remains difficult to probe the micrometer scaled areas of interest. Nevertheless, recent advances in synchrotron radiation have made it possible to probe structures locally in devices using micro- and nanobeams. ${ }^{17}$

\footnotetext{
${ }^{a)}$ Electronic mail: mathias.huss-hansen@fysik.dtu.dk

${ }^{b}$ Present address: Center for Nano Optics, University of Southern Denmark, 5230 Odense, Denmark.
}

In this letter, we use micro-focused $\mathrm{X}$-rays to investigate

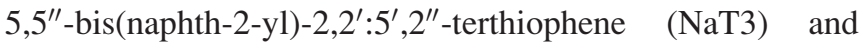
establish its structural fundamentals in OFETs with and without embedded silver nanoparticles.

Figure 1(a) shows the chemical structure of NaT3. The synthesis of NaT3 follows the Suzuki cross-coupling protocols reported in Ref. 9.

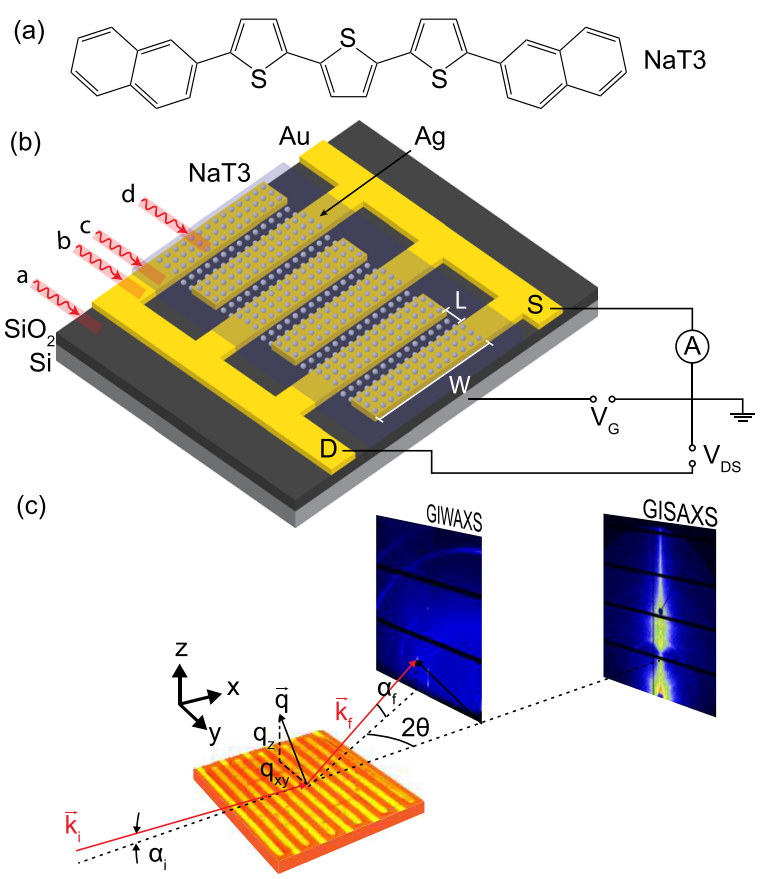

FIG. 1. (a) Chemical structure of NaT3. (b) Illustration of the OFET design with interdigitated gold drain (D) and source (S) electrodes. W and L are the width and length of the transistor channels, respectively. The positions of the $\mu$-beam corresponding to Figs. 3(a)-3(d) are indicated by wavy red lines. Not drawn to scale. (c) Illustration of the employed GIWAXS/GISAXS setup. 
Figure 1(b) shows the structure of the investigated OFETs fabricated by the following procedure, as described in Ref. 10. A highly n-doped $\mathrm{Si}$ substrate formed the back electrode with a $200 \mathrm{~nm}$ thick thermally grown $\mathrm{SiO}_{2}$ layer as a gate dielectric. The interdigitated source and drain electrodes were fabricated by E-beam evaporation of $30 \mathrm{~nm} \mathrm{Au}$ on top of $3 \mathrm{~nm}$ of Ti, structured by photolithography and lift-off in acetone. The transistor channel dimensions were $2 \mathrm{~mm} \times 10 \mu \mathrm{m}$ (width $\times$ length) corresponding to a width/length $(\mathrm{W} / \mathrm{L})$ ratio of 200:1. Ag nanoparticles with diameters of $\sim 40 \mathrm{~nm}$ were subsequently patterned in a nominal square lattice (period $=200 \mathrm{~nm}$, corresponding to a surface coverage of $\sim 3 \%$ ) on top of the gate dielectric and electrodes by E-beam lithography in poly (methyl methacrylate) (PMMA) followed by thermal evaporation of $40 \mathrm{~nm}$ of $\mathrm{Ag}$ on top of $3 \mathrm{~nm}$ Ti and lift-off in acetone. Finally, a $55 \mathrm{~nm}$ thick NaT3 layer was deposited atop the substrate by vacuum sublimation. The film thickness was determined by atomic force microscopy (AFM). Each substrate contained two transistors: one with and one without nanoparticles. This allowed direct comparison to investigate the influence of the nanoparticles on devices that had been processed in exactly the same manner.

Figure 1(c) illustrates the employed experimental setup. The devices were investigated by means of regular and microbeam ( $\mu$-beam) grazing incidence $\mathrm{X}$-ray scattering in wide- and small-angle configurations (GIWAXS/GISAXS). The measurements with a regular beam were carried out at XMaS (BM28) at the European Synchrotron Radiation Facility (ESRF), Grenoble. The X-ray energy was $10 \mathrm{keV}$, and the beam size $100 \mu \mathrm{m}$ $\times 260 \mu \mathrm{m}$ (vert. $\times$ hor.). The angle of incidence was $0.17^{\circ}$, slightly above the critical angle of NaT3 $\left(0.11^{\circ}\right)$, and the data were recorded using a MAR 165 CCD detector. The sample-to-detector distance was $330 \mathrm{~mm}$. The $\mu$-beam measurements were carried out at the MicroFocus end station at the P03 beamline (MiNaXS) at PETRA III, Hamburg. The X-ray energy was $13.19 \mathrm{keV}$, and the beam size was $16 \mu \mathrm{m} \times 22 \mu \mathrm{m}$ (vert. $\times$ hor.) at the sample. The employed incidence angle was $0.42^{\circ}$. The GIWAXS and GISAXS images were recorded separately without moving the sample using Pilatus $300 \mathrm{~K}$ and Pilatus $1 \mathrm{M}$ detectors, respectively. The sample-to-detector distances were $220 \mathrm{~mm}$ for GIWAXS and $5 \mathrm{~m}$ for GISAXS, calibrated by lanthanum hexaboride and silver behenate.

The GIWAXS images were transformed to the reciprocal space by reciprocal space mapping (RSM) using the Pygix python library and the fiber transformation originally described by Stribeck. ${ }^{18}$ This transformation is valid since the film exhibits fiber texture in the $x-y$ plane around the surface normal ( $z$-axis). The GISAXS images were converted to the $\mathbf{q}$ space using FitGISAXS ${ }^{19}$ The crystal grain-sizes were analyzed using Scherrer grain-size analysis adapted for grazing-incidence scattering with an area detector. ${ }^{20}$ In this procedure, the tangential full width half maximum (FWHM) of the RSM peak intensities was fitted with a Lorentzian distribution. By carrying out resolution analysis, the experimental contribution to the peak broadening (including smearing from the beam divergence, energy bandwidth, and the finite length of the beam footprint) was determined to be less than $10 \%$ of the total peak width and was therefore treated as negligible.

To obtain a qualitative understanding of the molecular packing inside the thin film unit cell, density functional theory (DFT) was employed using the GPAW package. ${ }^{21}$ The A Form packing reported in Ref. 8 was used as a starting point together with the experimentally found unit cell, and the atoms were allowed to relax until the atomic forces were below $1 \times 10^{-2} \mathrm{eV} / \AA$. The Bayesian error estimation functional $^{22}$ (BEEF-vdW) and projector-augmented wave potentials were used, with a plane-wave cutoff of $700 \mathrm{eV}$ and a converged $1 \times 6 \times 4$ Mockhorst-Pack grid.

The electric characterization of the OFETs was performed with a custom-built characterization set-up based on a LabVIEW-controlled data acquisition card connected to voltage and current amplifiers, providing the input, and a probe station measuring the output signal from the OFET. The gate voltage $\left(V_{G}\right)$ was swept from $0 \rightarrow-15 \mathrm{~V}$ with a constant drain-source voltage $V_{D S}=-15 \mathrm{~V}$.

Figure 2(a) shows a 2D GIWAXS pattern of the NaT3 thin film from the OFET by a regular beam. The pattern exhibits a series of vertically smeared peaks (Bragg rods) originating from the mosaicity of the NaT3 crystallites, with the molecules standing nearly vertically on the substrate. The smearing of the Bragg peaks along $\mathbf{q}_{z}$ suggests that NaT3 exhibits a higher degree of disorder along the stacking direction perpendicular to the surface normal compared with the closely related but symmetrical 5,5'-bis(naphth-2-yl)2,2'-bithiophene (NaT2), which shows equally resolved Bragg peaks (rather than rods) in both directions. ${ }^{7}$ Yet the pattern has a prominent similarity to what is reported for 5-decyl-5" -(naphthalen-2-yl)-2,2':5', $2^{\prime \prime}$-terthiophene (D3TN) in Ref. 23, which likewise contains three thiophene rings with one end-group replaced by an alkyl-group. The observed Bragg peaks can be consistently indexed assuming a monoclinic unit cell with parameters $a=25.7 \AA, b=5.87 \AA$, $c=8.03 \AA$, and $\beta=98.9^{\circ}$ and volume $V=1.20 \times 10^{4} \AA^{3}$.
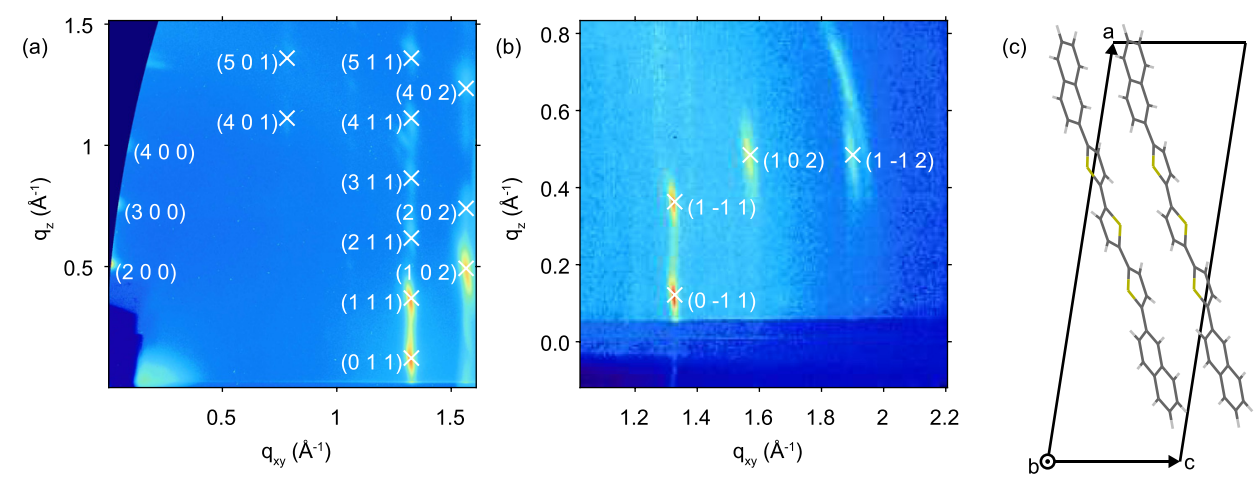

FIG. 2. GIWAXS pattern from (a) entire OFET device and (b) area of OFET with embedded nanoparticles, measured from the same sample. The Bragg peaks (and corresponding Miller indices) originate from the fibertextured NaT3 film crystallites. (c) Proposed NaT3 unit cell and molecular packing in OFETs. 

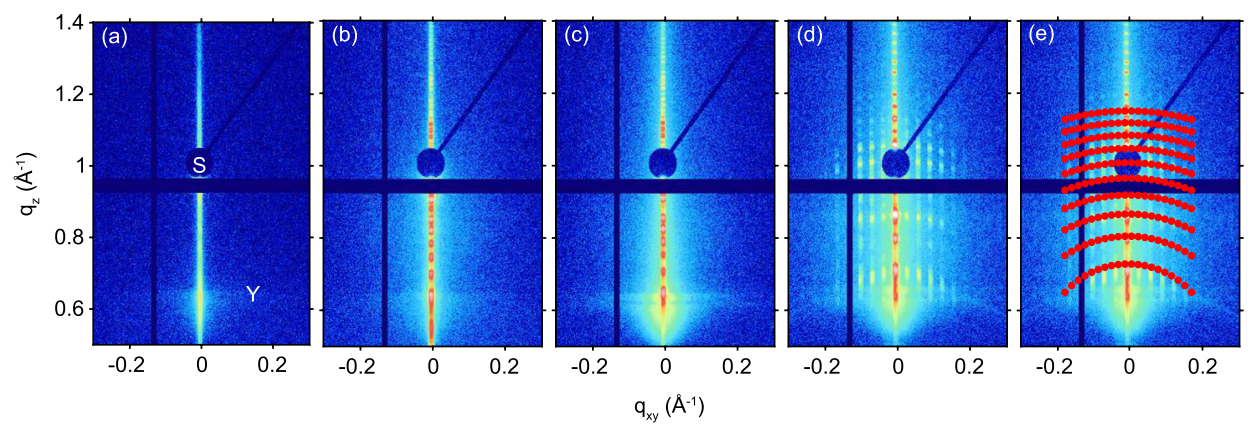

FIG. 3. GISAXS images from different areas of the OFET: (a) $\mathrm{SiO}_{2}$ substrate, (b) Au electrodes on $\mathrm{SiO}_{2}$, (c) $\mathrm{NaT} 3$ atop the electrodes, and (d) and (e) Ag nanoparticles embedded in NaT3 atop the electrodes [the corresponding positions of the $\mu$-beam are indicated in Fig. 1(b)]. S indicates the position of the specular beamstop; $\mathrm{Y}$ indicates the Yoneda peaks of $\mathrm{SiO}_{2}$ and $\mathrm{NaT3}$. The red dots indicate the calculated positions of the reflections from the interdigitated electrodes with a $10 \mu \mathrm{m}$ period.

Our unit cell choice follows the notation described in Ref. 7 with (100)-texture and standing molecules (defined with a as the long axis nearly along the backbone of the molecule). The unit cell observed in our thin film devices differs significantly from the unit cell reported for bulk NaT3 in Ref. 8 . The reported bulk unit cell has 6 molecules (and the volume $3.44 \times 10^{4} \AA$ ) , compared to $Z=2$ for the thin film case. This suggests that the long range order along the long axis is reduced for films.

Figure 2(b) shows a typical GIWAXS pattern from the area of the OFET with embedded particles measured by a $\mu$ beam. The data are consistently indexed with the same unit cell parameters, indicating that there are no significant changes in the NaT3 unit cell with and without embedded particles. The proposed molecular packing is shown in Fig. 2(c).

Table I compiles the determined crystal sizes for NaT3 in devices with and without embedded particles. There are no significant changes in the crystal sizes between these two cases within the given error bounds (calculated as one standard deviation from the least squares fitting). Interestingly, the typical crystal sizes of NaT3 in films are on the same order of magnitude as the nominal size of the embedded particles. It is therefore likely that the particles are incorporated into the mosaic crystal structure causing only local hindrance to the molecular growth. The opposite could be the case if the average crystallite size was much larger.

Figure 3 shows the GISAXS images from different areas of the device when the incident $\mathrm{X}$-ray was perpendicular to the interdigitated electrodes, with $\mathbf{q}_{z}$ and $\mathbf{q}_{x y}$ corresponding to the out-of-plane and in-plane wave vectors, respectively. The image from the bare $\mathrm{SiO}_{2}$ substrate [Fig. 3(a)] features a bright specular rod along $\mathbf{q}_{z}$ with the specular reflected beam covered by the beamstop (indicated with an $\mathrm{S}$ ). The specular beam is located at an exit angle equal to the incidence angle of $\alpha_{i}=0.42^{\circ}$. At $q_{z}=0.65$, the observed horizontal scattering feature is the so-called Yoneda peak (indicated with a Y) from

TABLE I. Crystal sizes of various crystallographic planes with their corresponding Miller indices.

\begin{tabular}{lcccc}
\hline \hline Sample & $\begin{array}{c}(0 \overline{1} 1) \\
(\mathrm{nm})\end{array}$ & $\begin{array}{c}(1 \overline{1} 1) \\
(\mathrm{nm})\end{array}$ & $\begin{array}{c}(102) \\
(\mathrm{nm})\end{array}$ & $\begin{array}{c}(1 \overline{1} 2) \\
(\mathrm{nm})\end{array}$ \\
\hline With nanoparticles & $28 \pm 2$ & $28 \pm 2$ & $19 \pm 1$ & $17 \pm 2$ \\
Without nanoparticles & $30 \pm 3$ & $33 \pm 3$ & $21 \pm 2$ & $22 \pm 2$ \\
\hline \hline
\end{tabular}

$\mathrm{SiO}_{2}$ that appears at the critical angle of the material $\left(\alpha_{c}=\right.$ $0.146^{\circ}$ for $\mathrm{SiO}_{2}$ ). On the image from the area with Au electrodes on top of $\mathrm{SiO}_{2}$ [Fig. 3(b)], multiple bright scattering peaks are found along the specular rod at $q_{x y}=0$. These peaks are consistent with the diffraction pattern from a 1D lateral grating formed by the Au electrodes, aligned perpendicular to the incoming beam. The same feature is present on the image from the area with NaT3 deposited on top of the electrodes [Fig. 3(c)] with small modulations due to reflection/refraction effects in the thin film overlayer. The Yoneda peak for NaT3 $\left(\alpha_{c}=0.11^{\circ}\right)$ appears at $q_{z}=0.61$, just below the Yoneda peak of $\mathrm{SiO}_{2}$. Furthermore, there is an increase in diffuse scattering from the disorder of the thin film structure.

Figure 3(d) shows the image from the area with Ag particles embedded in NaT3 on top of the interdigitated $\mathrm{Au}$ electrodes. The scattering intensity in the in-plane $\mathbf{q}_{x y}$ direction is dominated by a series of sharp peaks that appear to be correlated with the peaks found along the specular rod and are located on arcs extending from $q_{x y}=0$. The high scattering order and sharpness of peaks found along $\mathbf{q}_{x y}$ point to a high correlation between particles, and due to the large footprint of the beam, it is inferred that the particles are wellaligned across the device. From the peak spacing, the average interparticle distance can be derived as $d_{\text {particle }}=2 \pi / \Delta q_{x y}$, leading to a distance of $198 \pm 2 \mathrm{~nm}$. The peaks found along the specular rod are spaced further apart than in Figs. 3(b) and 3(c) due to the electrode spacing being halved in the area, where the electrodes are interdigitated. The diffraction from a one-dimensional grating perpendicular to the incoming beam can be written as

$$
\cos \left(\alpha_{f}\right)-\cos \left(\alpha_{i}\right) \cos (2 \theta)=\frac{m \lambda}{d},
$$

where $\alpha_{i}$ and $\alpha_{f}$ are the incidence and exit angles, $2 \theta$ is the in-plane scattering angle, $m$ is the order of diffraction, $\lambda$ is the X-ray wavelength, and $d$ is the grating period. The observed scattering patterns correspond to the computed diffraction peaks for a grating with $d=10 \mu \mathrm{m}$ [red dots in Fig. 3(e)]. Here, the diffraction patterns are shown for $\mathrm{m}=-5$ to 4 for 20 equally spaced values of $2 \theta$ in the range of $\pm 0.2^{\circ}$.

Figure 4 shows the transfer curves of the OFET with and without embedded particles. The saturation mobilities are extracted from the slopes of the $I_{D S}^{-1 / 2}$ vs $V_{G}$ curves (not shown) in the high bias regime between $V_{G}=-10$ and -15 . 


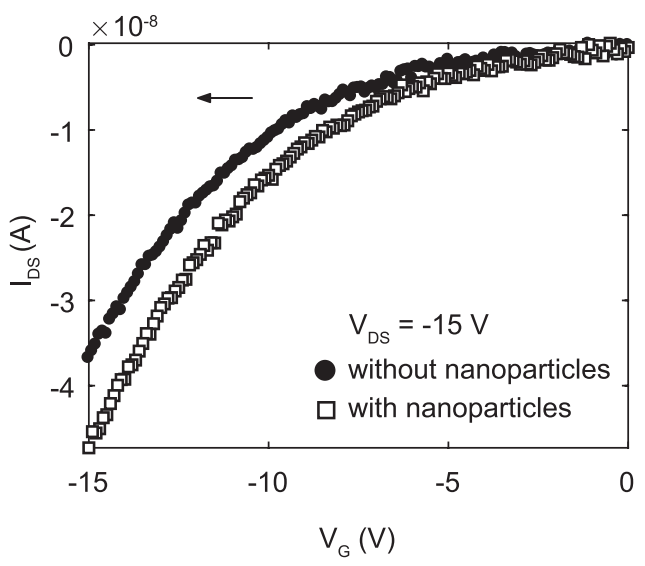

FIG. 4. Transfer curves of NaT3 OFETs with and without embedded particles. The arrow indicates the sweeping direction.

TABLE II. Saturated mobilities of OFETs with and without particles. Reported values are in $\times 10^{-4} \mathrm{~cm}^{2} /(\mathrm{V} \mathrm{s})$.

\begin{tabular}{lccccc}
\hline \hline Sample & 1 & 2 & 3 & 4 & Mean \\
\hline With nanoparticles & 0.38 & 1.09 & 2.02 & 1.40 & $1.2 \pm 0.59$ \\
Without nanoparticles & 0.41 & 0.69 & 1.77 & 1.56 & $1.1 \pm 0.57$ \\
\hline \hline
\end{tabular}

The saturation mobilities from 8 devices are compiled in Table II. The mean value for devices with embedded particles is $1.2 \times 10^{-4} \mathrm{~cm}^{2} /(\mathrm{V} \mathrm{s})$ compared to $1.1 \times 10^{-4} \mathrm{~cm}^{2} /$ (Vs) for devices without the embedded particles. The two results lie within the range of uncertainty, indicating that the particles do not notably affect the field-effect mobility of the device. Since the charge transport in OFETs occurs near the interface between the semiconductor and the gate dielectric, where the particles are also positioned, we expect that the particles would influence the FET performance. However, this is not observed. This could be due to the fact that the particles only occupy $\sim 3 \%$ of the channel area and that a noticeable change in performance would only be visible if a larger fraction of the channel area was covered.

In conclusion, we have found that the NaT3 thin film unit cell differs significantly from the one reported for the bulk, with a decreased long range order and only two molecules per unit cell. When E-beam deposited silver particles are embedded in the OFET device structure, there is no further change in the NaT3 unit cell or apparent crystal sizes. The measured field-effect mobility in the saturated regime averaged as $1.2 \times 10^{-4} \mathrm{~cm}^{2} /(\mathrm{V} \mathrm{s})$ for OFETs with the particles compared to $1.1 \times 10^{-4} \mathrm{~cm}^{2} /(\mathrm{V} \mathrm{s})$ for devices without particles. These results form a structural base for designing field-effect transistors with aryl end-capped oligothiophenes.

The authors thank A. Osadnik and A. Lützen of Univ. Bonn for providing NaT3, O. Bikondoa of ESRF and M. Schwartzkopf of Petra III for experimental assistance, M. Torkkeli of Technical Univ. Denmark and M. J. Winokur of Univ. Wisconsin-Madison for discussions, and DANSCATT for financial support.

${ }^{1}$ H. Sirringhaus, Adv. Mater. 26, 1319 (2014).

${ }^{2}$ C. Zhang, P. Chen, and W. Hu, Chem. Soc. Rev. 44, 2087 (2015).

${ }^{3}$ Y. Zang, F. Zhang, D. Huang, X. Gao, C. Di, and D. Zhu, Nat. Commun. 6, 6269 (2015).

${ }^{4}$ K.-J. Baeg, M. Binda, D. Natali, M. Caironi, and Y.-Y. Noh, Adv. Mater. 25, 4267 (2013).

${ }^{5}$ A. N. Aleshin, J. Y. Lee, S. W. Chu, J. S. Kim, and Y. W. Park, Appl. Phys. Lett. 84, 5383 (2004).

${ }^{6}$ H. Tian, Y. Chen, W. Li, D. Yan, Y. Geng, and F. Wang, Org. Electron. 15, 1088 (2014).

${ }^{7}$ M. K. Huss-Hansen, A. E. Lauritzen, O. Bikondoa, M. Torkkeli, L. Tavares, M. Knaapila, and J. Kjelstrup-Hansen, Org. Electron. 49, 375 (2017).

${ }^{8}$ H. Tian, J. Shi, B. He, N. Hu, S. Dong, D. Yan, J. Zhang, Y. Geng, and F. Wang, Adv. Funct. Mater. 17, 1940 (2007).

${ }^{9}$ F. Balzer, M. Schiek, A. Osadnik, I. Wallmann, J. Parisi, H.-G. Rubahn, and A. Lützen, Phys. Chem. Chem. Phys. 16, 5747 (2014).

${ }^{10}$ J. Linnet, A. R. Walther, O. Albrektsen, L. Tavares, R. L. Eriksen, P. B. W. Jensen, A. Osadnik, S. Hassing, A. Lützen, and J. Kjelstrup-Hansen, Org. Electron. 46, 270 (2017).

${ }^{11}$ A. D. Carlo, F. Piacenza, A. Bolognesi, B. Stadlober, and H. Maresch, Appl. Phys. Lett. 86, 263501 (2005).

${ }^{12}$ A. E. Lauritzen, M. Torkkeli, O. Bikondoa, J. Linnet, L. Tavares, J. KjelstrupHansen, and M. Knaapila, Langmuir 34, 6727 (2018).

${ }^{13}$ X. Liu, L. Tavares, A. Osadnik, J. L. Lausen, J. Kongsted, A. Lützen, H.G. Rubahn, and J. Kjelstrup-Hansen, Org. Electron. 15, 1273 (2014).

${ }^{14}$ D. M. DeLongchamp, R. J. Kline, D. A. Fischer, L. J. Richter, and M. F. Toney, Adv. Mater. 23, 319 (2010).

${ }^{15}$ H. Yoshida, K. Inaba, and N. Sato, Appl. Phys. Lett. 90, 181930 (2007).

${ }^{16}$ J.-H. Kim, Y.-N. Kwon, E. Lee, J. Y. Jung, J.-Y. Kim, J. Shin, J.-I. Park, and A. Choi, Appl. Phys. Lett. 111, 233301 (2017).

${ }^{17}$ M. Ruderer, V. Körstgens, E. Metwalli, M. Al-Hussein, U. Vainio, S. Roth, R. Döhrmann, R. Gehrke, R. Gebhardt, M. Burghammer, and P. Müller-Buschbaum, Nucl. Instrum. Methods Phys. Res., Sect. B 268, 403 (2010).

${ }^{18}$ N. Stribeck and U. Nöchel, J. Appl. Crystallogr. 42, 295 (2009).

${ }^{19}$ D. Babonneau, J. Appl. Crystallogr. 43, 929 (2010).

${ }^{20}$ D.-M. Smilgies, J. Appl. Crystallogr. 42, 1030 (2009).

${ }^{21}$ J. J. Mortensen, L. B. Hansen, and K. W. Jacobsen, Phys. Rev. B 71, 035109 (2005).

${ }^{22}$ J. Wellendorff, K. T. Lundgaard, A. Møgelhøj, V. Petzold, D. D. Landis, J. K. Nørskov, T. Bligaard, and K. W. Jacobsen, Phys. Rev. B 85, 235149 (2012).

${ }^{23}$ T. K. An, S. H. Jang, S.-O. Kim, J. Jang, J. Hwang, H. Cha, Y. R. Noh, S. B. Yoon, Y. J. Yoon, L. H. Kim, D. S. Chung, S.-K. Kwon, Y.-H. Kim, S.-G. Lee, and C. E. Park, Chem. Eur. J. 19, 14052 (2013). 